\title{
Transient Analysis of Production Inventory System with Different Rates of Production and Random Switching Time
}

\author{
Mohammad Ekramol Islam ${ }^{1}$, Md. Sharif Uddin² and Mohammad Ataullah ${ }^{3 *}$ \\ ${ }^{1}$ Department of Business Administration, Northern University Bangladesh.Dhaka-1205 \\ ${ }^{2}$ Department of Mathematics, Jahangirnagar University, Bangladesh, Dhaka-1342 \\ 3,* Assistant professor of Mathematics, Directorate of Secondary and Higher Education, \\ Ministry of Education, Bangladesh, Dhaka-1000
}

\begin{abstract}
This paper analyzes a single product stochastic inventory model with two different production rates where demand follows Poisson distribution and product has a finite lifetime which is level dependent. It is assumed that the system is reached on a predetermined level, the system is converted to ON mode from OFF mode with a significant switching time with an exponential parameter. During switching time no demand will be served, the demand during switching time is lost forever. Here backlogs are allowed and during backlogs, the Production rate is higher than that of normal production time. We assume that the product will decay for the time under consideration. Some system characteristics are displayed with time variation.
\end{abstract}

Keywords: Product Inventory, Stochastic Process, Poisson distribution, Product Life Time, Switching Time, Backlogs, Level Dependent, Exponential Process.

\section{INTRODUCTION}

Inventory control is one the most important criteria for supply chain management. The analysis of the inventory model include product deterioration characteristic. In several existing models, it is assumed that products have infinite shelf time but in real life situations, a certain amount of decay or waste is experienced on the stocked items. Deterioration of items in the inventory system occurred due to presence of some factors lime storage, weather condition including the nature of the particular product under study; out of them, some may be perishable whereas others can be stored for an indefinite period without being perished. The deterioration is usually a function of the total amount of inventory on hand. Hence, the need to study the perishability characteristic of the inventory system.

\section{LITERATURE REVIEW}

Many researchers in the field have already done an extensive research work by assuming a constant rate of decay and constant demand, but use a deterministic approach that does not take into consideration the uncertainty of demand. We add uncertainty by including a new variable that reflects the probability of different scenarios. Ghare and Schrander [3] first pointed out the effect of decay in inventory analysis. However, they studied a simple Economic Order Quantity (EOQ) model the exponential decay. Krishnamoorthy and Mohammad Ekramol Islam[4], Present a product inventory model with retrial of customers, M. Sharafali [9]considered a one-product, one-machine production-inventory problem operating under an (s, S) policy. The production facility may be set up and in production, shut down and idle or failed and under repair. Back-order is not permitted. By identifying a semiregenerative process, expressions for the probability mass function for the inventory process at any time and in the stationary regime are obtained. Nahmias [10] provided a reference list of 77 periodicals and books dealing with the ordering policies for perishable inventories. Feng-Tsung Cheng et al. [2], investigates the optimal inventory replenishment policy for an economic production quantity (EPQ) model with backordering, rework and machine

breakdown taking place in stock piling time. According to Chiu et al. [12] very little investigation was done on production inventory in the past with stochastic demand. Most of those cases switching time was neglected to count. But in the practical cases there always needs some specific time to change the mode of production from OFF mode to ON mode. If switching time is 
fixed then there is nothing to worry, but in most of the cases, it cannot be exactly estimated well in advance. This switching time may follow some probability distribution. Generally, the analysis becomes highly complex when the processes are stochastic.

M. Berg et al. [5] noticed that in the production-inventory systems a number of producing machines are susceptible to failure following which must be repaired to make them operative again. The machines' production can also be stopped deliberately due to stocking capacity limitations or any other relevant considerations. The interplay between the processes involved, namely, production, demand, and failure/repair or reliability, in conjunction with the shutdown policy used, determine the inventory accumulation process and possible shortages. Mohammad Ekramol Islam et al. [8] presented an inventory system with postponed demands considering reneging pools and rejecting Buffer's customers. B.C. Giri and T. Dohi [1] presented an exact formulation of stochastic EMQ model for an unreliable production system under a general framework in which the time to machine failure, corrective (emergency) and preventive (regular) repair times are assumed to be random variables. For exact financial implications of the lot-sizing decisions, the EMQ model is formulated based on the net present value (NPV) approach. Then, by taking limitation on the discount rate, the traditional long-run average cost model is obtained. S. K. Goyal and B. C. Giri [11] presented an inventory model with exponential declining demand and constant deterioration is considered. The time-varying holding cost is a linear function of time. Shortages are not allowed. The items (like food grains, fashion apparels and electronic equipment's) have fixed shelf-life which decreases with time during the end of the season. Mohammad Ekramol Islam [6], studied a Product inventory model for deteriorating items with various product rates and constant Demand. This work is the extension of the research work of Mohammad Ekramol Islam et al. [7], presented a perishable stochastic inventory system with different rates of production and random switching time.

\section{ASSUMPTIONS AND NOTATIONS}

\section{a) Assumptions}

i. Two production rates are considered where production rate is higher during backlogs than normal time

ii. When inventory level reaches at a predetermine level $-\mathrm{N}$, the system converted to ON mode from OFF mode with parameter which follows exponential distribution.

iii. Perishability is time dependent.

iv. During switching time, no demand allow, so demand at the switching time is lost forever.

v. If inventory level reaches at order level, production process will switch off.

\section{b) Notations}

i. $\lambda \rightarrow$ Arrival rate,

ii. $\quad \mu \rightarrow$ Production rate during backlogs,

iii. $\delta \rightarrow$ Normal production rate,

iv. $\mathrm{S} \rightarrow$ Maximum inventory level,

v. $\rho \rightarrow$ The decay rate of items,

vi. $\quad \tau \rightarrow$ Switching time

vii. $\quad I(t)$ Inventory level at time $t$,

viii. $E \rightarrow E_{1} \cup E_{2}$ is the state space of the process where, $E_{1}=\{(t, 0): t=-N+1, \ldots, S\}$ and

$$
E_{2}=\{(t, 1): t=-N+1, \ldots, S-1\}
$$

\section{MODEL ANALYSES}

In this model, we assume that the inventory level $\mathrm{S}$ at the starting point and the system is in OFF mode. Demand follows Poisson distribution with rate $\lambda$. Inventory level depletes due to customers and items will decay with time passes, which is level dependent. When inventory level reaches to the level $-\mathrm{N}$ then the system will switched $\mathrm{ON}$ mode. In the inventory system, inventory level $I(t)$ takes the value

$$
A=\{-N,-N+1, \ldots, 0,1,2, \ldots, S\}
$$

To get a two dimensional Markov process we introduce the $\{X(t), t \geq 0\}$

Where, $X(t)$ is defined by

$$
X(t)= \begin{cases}1 & \text { when production is } O N \\ 0 & \text { whwn production is } O F F\end{cases}
$$

Now, the infinitesimal generator of the two dimensional Markov process $\{I(t), X(t) ; t \geq 0\}$ is defined on the state space E. it is noted that the Markov process is a pure birth and death process during the transition from the state $(S, 0)$ through the state $(S-1,0), \ldots,(-N+1,0)$ when the production process is in OFF mode. When inventory level in the state $(-N, 0)$ then the 
system is switched ON. Switching time follows exponential distribution with parameter $\alpha$ and reached the state $(N, 1)$ from $(-N, 0)$. From this state onward the process till it reaches the level $(S, 0)$.

Let us assumed $I(0)=S$ and $X(0)=0$. Let us consider the transition probabilities:

$$
P_{(S, 0)(i, j)}(t)=P\{I(t), X(t)=(i, j) \mid I(0), X(0)=(S, 0)\}
$$

From now onwards we can write

$$
P_{(i, j)}(t) \text { for } P_{(s, 0)(i, j)}(t)
$$

Kolmogorov difference differential equations for the system $P_{(i, j)}(t)$ are given bellow:

When system is OFF mode:

$P^{\prime}{ }_{(s, 0)}(t)=-(\lambda+S \rho) P_{(s, 0)}(t)+\delta P_{(s-1,1)}(t) \ldots(\mathrm{i})$

$P_{(i, 0)}^{\prime}(t)=-(\lambda+i \rho) P_{(i, 0)}(t)+(\lambda+(i+1) \rho) P_{(i+1,0)}(t) ; i=S-1, \ldots, 0 \quad \ldots \quad$ (ii)

$P^{\prime}{ }_{(i, 0)}(t)=-\lambda P_{(i, 0)}(t)+\lambda P_{(i+1,0)}(t) ; i=-1, \ldots,-N+1 \quad \ldots \quad$ (iii)

$P^{\prime}{ }_{(-N, 0)}(t)=-\tau P_{(-N, 0)}(t)+\lambda P_{(-N+1,0)}(t) \quad \ldots \quad$ (iv)

When the system is ON mode:

$P^{\prime}{ }_{(s-1,1)}(t)=-(\lambda+i \rho+\delta) P_{(s-1,1)}(t)+(\lambda+i \rho) P_{(s, 0)}(t) \ldots(\mathrm{v})$

$P^{\prime}{ }_{(i, 1)}(t)=-(\lambda+i \rho+\delta) P_{(i, 1)}(t)+(\lambda+(i+1) \rho) P_{(i+1,0)}(t)+\delta P_{(i+1,1)}(t) ; \mathrm{i}=\mathrm{S}-2, \ldots, 0 \ldots \quad(\mathrm{vi})$

$P^{\prime}{ }_{(i, 1)}(t)=-(\lambda+\mu) P_{(i, 1)}(t)+\lambda P_{(i+1,1)}(t)+\mu P_{(i+1,1)}(t) ; \mathrm{i}=-\mathrm{N}+1, \ldots,-2,-1 \quad \ldots \quad$ (vii)

$P_{(-N, 1)}^{\prime}(t)=-\mu P_{(-N, 1)}(t)+\tau P_{(-N, 0)}(t)+\lambda P_{(-N+1,1)}(t) \quad \ldots \quad$ (viii)

We solve this system of ordinary differential equations by using the Runge-Kutta method of fourth order based on specific parameters. We have plotted the graphs based on ODE's and performance measures. The effect of various parameters on the system performance measures such as expected number of customers in the system and mean waiting time in the system are studied. MATLAB R2016a software is used to develop the computational program.

\section{SOME PERFORMANCE MEASURES}

a) Mean inventory level in the system

Let the expected inventory level

$$
L s(t)=\sum_{i=1}^{S} i P_{(i, 0)}+\sum_{i=1}^{S-1} i P_{(i, 1)}
$$

b) Expected number of perishable items

Let the number of perishable items

$$
L p(t)=\sum_{i=1}^{S} i \rho P_{(i, 0)}+\sum_{i=1}^{S-1} i \rho P_{(i, 1)}
$$

c) Expected backlogs in the system

Let the expected backlogs

$$
L b(t)=\sum_{i=-N}^{-1}|i| P_{(i, 0)}+\sum_{i=-N}^{-1}|i| P_{(i, 1)}
$$

d) Expected number of customer lost

Let expected number of customer lost $C L(t)=\lambda P_{(-N, 0)}+\lambda P_{(-N, 1)}+\alpha \lambda P_{(-N, 0)}$

e) Expected switching time $\alpha$.

f) Expected total cost of the system

$$
E T C(t))=L+c_{1} L s(t)+c_{2} L p(t)+c_{3} N b(t)+c_{4} C L(t) .
$$

\section{NUMERICAL RESULTS AND DISCUSSION}

In all numerical computations, the model parameters are taken as

$S=3, N=2, \lambda=1, \alpha=0.21, \mu=3, \delta=2, \mathrm{~L}=25, c_{1}=0.15, c_{2}=0.25, c_{3}=0.1, c_{4}=0.35$. 
International Journal of Advances in Scientific Research and Engineering (ijasre), Vol 5 (1), January-2019

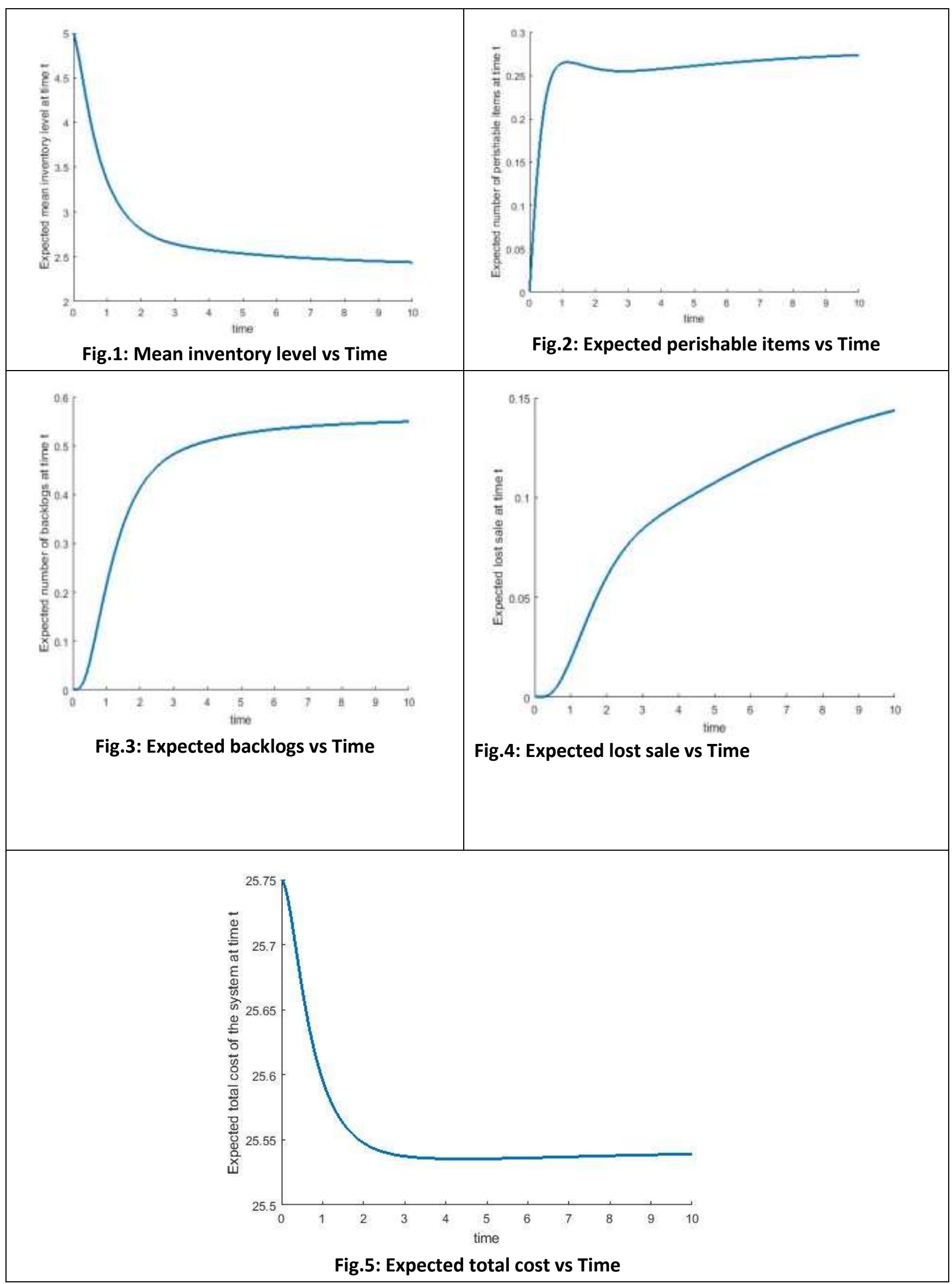




\subsection{Numerical Results and Discussion}

Numerical outcomes have been gotten by applying Runge-kutta fourth request technique in the arrangement of standard differential conditions (I) through viii) with the assistance of computational programming MATLAB R2016a for the parametric qualities steady with time interim $0 \leq \mathrm{t} \leq 10$ as appeared in the diagrams. Diagrams of different parameters versus time have been demonstrated the fig. 1 through fig. 5 .

Fig.1 investigates that the stock dimension diminished with time yet for long time interim it is decrease rate lower than from beginning stage. Fig. 2 demonstrates that the quantity of transient things raises quickly from starting and after some time passing diminishes perishability rate. Fib.3 Explain the quantity of overabundances with time variety. The figure demonstrates that average number of clients in the framework increment at the beginning stage however after some time, as creation rate is higher amid excesses period than that of ordinary generation day and age for higher estimation of generation rate gives lesser number of clients in the framework which is very sensible. From Fig.4 we see that the rate of lost deal is nearly low, which is reason by the way that underlying likelihood has been lessened essentially because of which some the probabilities have their qualities zero after some time which implies that probabilities are not relying on time just but rather upon different parameters as well. Fib.5 exhibits the normal aggregate expense of the framework, which demonstrate that at first aggregate expense, is higher than whatever remains of era considered in the model.

\section{CONCLUSION}

Investigation of single item inventory model under time subordinate entry and administration rates has been made .Under the examination the numerical outcomes for different execution measures have been acquired by utilizing Runge-kutta fourth request strategy with MATLAB R2016a. Our model can be considered under various servers' arrangement which may give progressively broad arrangement under time subordinate circumstances in order to make the model increasingly reasonable.

\section{REFERENCES}

1. Chiu, Y.S.P., C-K. Ting, C-B. Cheng, and F-Y. Lin, "Alternative approach for realistic lot-sizeproblem with random scrap rate and backlogging”, WSEAS Transactions on Mathematics, Vol. 6 (6), 2007, pp.736-740

2. Feng-Tsung Cheng, Huei-Hsin Chang and Singa Wang Chiu, "Economic Production Quantity Model with Backordering, Rework and Machine Failure Taking Place in Stock Piling Time", Wseas Transactions On Information Science And Applications, Issue 4, Volume 7, April 2010, ISSN: 1790-0832, p.p. 463-473

3. Ghare, P.N. and Schrader, G.F., "A Model for Exponentially Decaying Inventories", The Journal of Industrial Engineering, 15,1963, pp. 238-243.

4. Giri, B.C. and T. Dohi, "Exact formulation of stochastic EMQ model for an unreliable production system", Journal of the Operational Research Society, Vol. 56(5), 2005, pp.563-575.

5. Krishnamoorthy and Mohammad Ekramol Islam, "Product inventory model with retrial of customers", Journal of Stochastic modeling and Application, 06, 2003, pp.1-11.

6. M. Berg, M.J.M. Posner and H. Zhao, "Production inventory system with unreliable machines" Operation research, Vol. 42, No.1, 1994, p.p.111-118.

7. Mohammad Ekramol Islam, "Product inventory model for deteriorating items with various product rates and constant Demand", Proceedings of annual conference of Kerala mathematical association, January 8-10, 2004, p.p.p.15-23.

8. Mohammad Ekramol Islam, Mohammad Shahjahan Miah and A.B.M.AbdusSubhan Miah, "A perishable stochastic inventory models with different rates of production with backlogs and random switching time", Journal of National Academy of Science, Vol.31, No. 2, 2007, pp. 231-238.

9. Mohammad Ekramol Islam, K.M. Safiqul Islam, M Sharif Uddin, "Inventory system with postponed demands considering reneging pool's and rejecting Buffer's customers", International Conference on Mechanical, Industrial and Materials Engineering (ICMIME 2013), Paper ID: IE-01, 1-3 November, 2013, RUET, Rajshahi, Bangladesh.

10. M. Sharafali, “On a continuous review production inventory problem”, Operation Research Letter, 3,1984, $199-204$.

11. Nahmias, "Perishable inventory theory; A review", Operation research, 30,1982, 680-7-8,

12. S. K. Goyal and B. C. Giri, "Recent trends in modeling of deteriorating inventory", European Journal of Operational Research, vol.134, no. 1, 2001, pp. 1-16. 


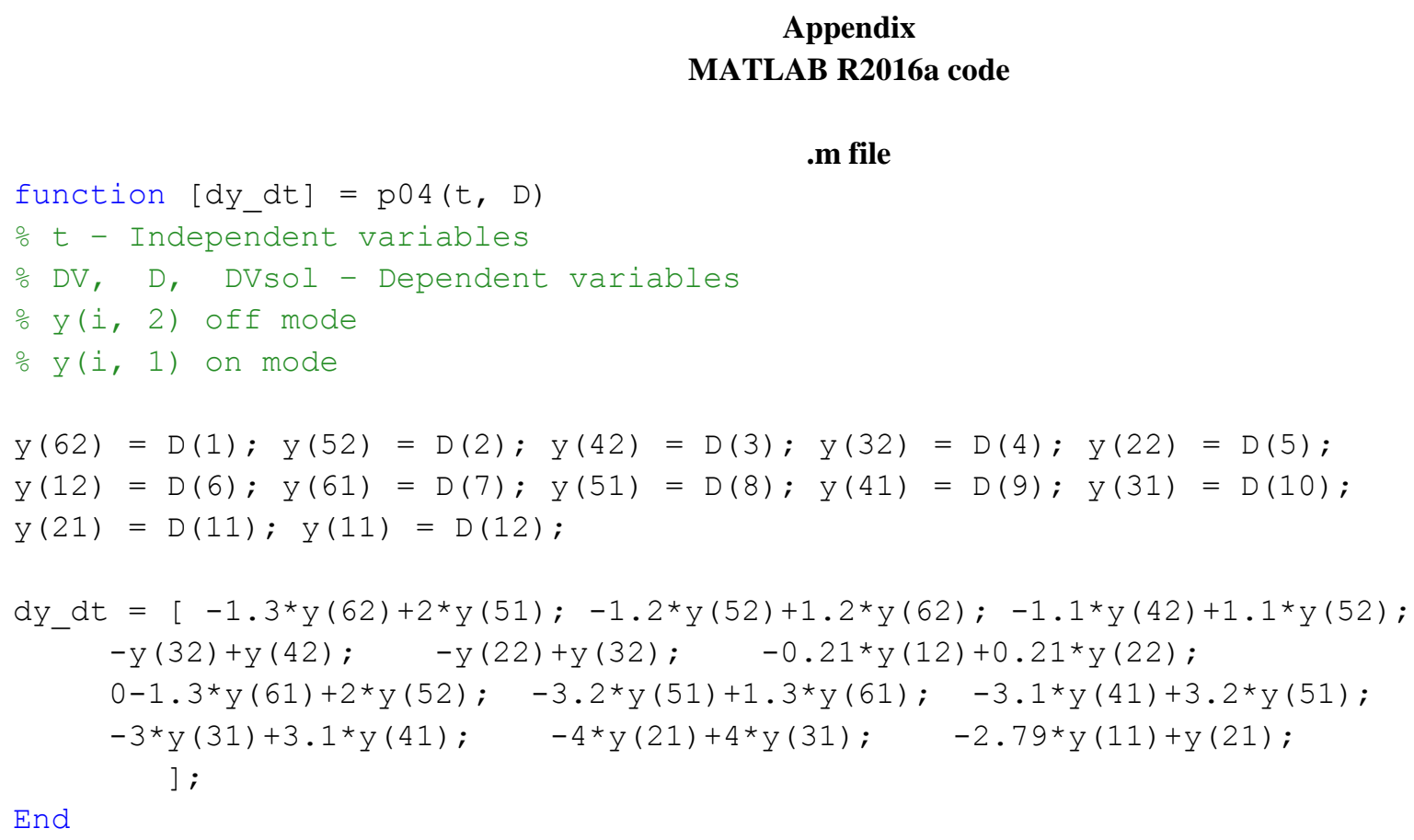

End

clearall; close all; clc

Scriptfile.m

$t=0: 0.5: 100$;

$i c 1=1 ; i c 2=0 ; i c 3=0 ; i c 4=0 ; i c 5=0 ; i c 6=0 ; i c 7=0 ; i c 8=0 ; i c 9=0 ; i c 10=0 ; i c 11=0 ; i c 12=0$;

$y 0=[i c 1, i c 2, i c 3, i c 4, i c 5, i c 6, i c 7, i c 8, i c 9, i c 10, i c 11, i c 12]$;

$\mathrm{L}=25$;

$[t, \mathrm{Dv}]=\operatorname{ode} 45(\mathrm{p} 04$ ', $t, \mathrm{y} 0) ;$

$\mathrm{A}=(\mathrm{DV})$;

$a=\operatorname{diag}(1 . / \operatorname{sum}(A, 2)) * A ;$

$\operatorname{MIL}=(\mathrm{a}(:, 11)+\mathrm{a}(:, 5))+2 *(\mathrm{a}(:, 4)+\mathrm{a}(:, 10))+3 *(\mathrm{a}(:, 3)+\mathrm{a}(:, 9))+4 *(\mathrm{a}(:, 2)+\mathrm{a}(:, 8))+5 *(\mathrm{a}(:, 1)+\mathrm{a}$ $(:, 7))$; 